\title{
La educación sexual y de género vs. el maltrato en la pareja. Escenario sobre la violencia en jóvenes de Baja California*
}

\section{Sex education and gender vs. abuse in relationships. Scenario on violence in young people in Baja California} Teresa Fernández de Juan**

\section{Resumen}

La violencia en las relaciones de noviazgo entre los jóvenes presenta manifestaciones crecientes a escala internacional y nacional, al igual que ocurre con el aumento de las enfermedades de transmisión sexual, el viH/ sida y el embarazo entre las adolescentes. En este trabajo se enfatiza por qué la principal estrategia para la prevención de ambas conductas de riesgo está íntimamente relacionada con la implementación de una eficiente, desprejuiciada y oportuna educación sexual, sobre todo en el ámbito escolar y con una perspectiva de género, de la que se carece en sentido general, en el país y en Baja California en particular.

Palabras clave: adolescencia, educación sexual, violencia de pareja, género, violencia en Baja California.

\begin{abstract}
Violence in the relationships of courtship among young people presents growing demonstrations at international and national levels, as it occurs with the increase in the diseases of HIV/AIDS, adolescent pregnancy and sexual transmission. This work emphasizes the main strategy for the prevention of both risk behaviour is directly related to the implementation of an efficient, unprejudiced and timely sex education, especially in schools, with a gender perspective, which is lacking in general sense, at home and in Baja California in particular.

Keywords: adolescence, sex education, intimate partner violence, gender violence, violence in Baja California.
\end{abstract}

Recibido el 15 de abril de 2013.

Aprobado el 20 de diciembre de 2013.

* La autora quiere dejar constancia de su agradecimiento a Juan Manuel Ávalos, Técnico Académico de Investigación de El Colegio de la Frontera Norte, por su invaluable apoyo a lo largo de la búsqueda y conformación de todos los datos del presente trabajo.

** Departamento de Estudios Culturales. El Colegio de la Frontera Norte. Correo electrónico: teresaf@colef.mx. 


\section{Introducción}

En 1873, el activista Anthony Comstok (como se citó en De Grazia, 1992) comparó la educación sexual con la pornografía satánica, iniciando una feroz campaña para lograr "la supresión del vicio" y así "salvar a los jóvenes de ellos mismos", hasta lograr que el Congreso de Estados Unidos aprobara la Ley Comstok. Esta ley permitía censurar y declarar ilegal la entrega o el transporte de cualquier tipo de información médica, anatómica o relativa al control de la natalidad y a las enfermedades, por englobarse dentro de lo que él mismo denominó "literatura vil y demoniaca" y material "obsceno o lascivo". Durante 40 años batalló contra la educación sexual hasta un nivel tan elevado que hostigó a doctores y escritores (como es el caso de Ida Craddock [cf. De Grazia, 1992]) llevando (según sus propias palabras) a 15 de ellos hasta el mismo suicidio. No es sino hasta 40 años después, en 1937, que esta ley es anulada y comienza a resquebrajarse en Nueva York este clima de terror y acoso.

Con estos antecedentes que parecerían rezagos feudales, sería inconcebible que el Dr. Álvarez-Gayou, Director del Instituto Mexicano de Sexología, puntualizara en este siglo y con gran preocupación, que "la educación sexual en México es prácticamente nula” y la gravedad de que "sea todavía un tema tabú que suscite temores y recelos, lo cual prevalece en la formación de quienes tienen a su cargo la preparación de las futuras generaciones: los maestros (en cualquier nivel de educación), por lo que nuestro proceso de enseñanza-aprendizaje carece hoy en día aún de una estructura con estos contenidos en sus programas" (citado por Serrano, 2013).

Como bien mencionara Gayle Rubin: "Las consecuencias de este gran paroxismo moral del siglo xIx perviven todavía. Han dejado una profunda huella en las actitudes sobre el sexo, en la práctica médica, en la educación infantil, en las preocupaciones de los padres, en la conducta de la policía y en las leyes sobre el sexo" (Rubin, 1989, p. 114).

A la postre, en quienes más influye negativamente esto es en los propios jóvenes. Desde el punto de vista de su salud física, el desconocimiento los expone a una enorme cantidad de riesgos, con el cambio frecuente de parejas, sin responsabilidad ni uso de medidas preventivas y el aumento 
de embarazos adolescentes (Saavedra y Bravo, 2009; Kendall, 2009; González y Vega, 2013). ${ }^{1}$

Y el otro aspecto, no menos alarmante, reside en la creciente violencia de pareja que, debido a la falta de educación en este tema, ya ni es reconocida como tal por los adolescentes. Que haya llegado a convertirse en algo invisible debido a su naturalización (Juárez y Gayet, 2005, p. 183) es doblemente alarmante, ya que si partimos de que la juventud mexicana representa cerca de la quinta parte de la población del país (20.2 millones de jóvenes entre 15 y 24 años de edad [Conapo, 2010]) y, por otro lado, ésta es una etapa de definiciones que impactará en los años subsiguientes, de no ser esta situación velozmente intervenida se proyectará cada vez más en la vida adulta de las parejas (Zamudio, Ayala y Andrade, 2011).

Basados además en los planteamientos de Garcés Fierros ${ }^{2}$ respecto de que la relevancia de este grupo poblacional radica no solamente en su gran volumen, sino "en el enorme desafío que plantean a la sociedad y al gobierno de garantizar la satisfacción de sus necesidades y el pleno desarrollo de sus capacidades" (Conapo, 2007, p. 1), por lo que invertir en ellos e incluirlos en estrategias diseñadas para su propio desarrollo, supone "contribuir a identificar escenarios susceptibles de toda intervención pública que tienda a fortalecer e incrementar sus capacidades en aras de su propio desarrollo y el de la sociedad en su conjunto"3 (Conapo, 2010, p. 9). Y siendo reconocida en el Fondo de Población de las Naciones Unidas en México la necesidad de que éstos adquirieran una información adecuada acerca de su sexualidad, debido a que comienzan su vida activa con tan escasos conocimientos del tema que ello ha provocado situaciones alarmantes (Conapo, 2007, p. 3), el presente artículo persigue el objetivo de contribuir a los esfuerzos por concientizar la necesidad de un cambio inmediato

${ }^{1}$ De hecho, Castellanos, miembro del Grupo Asesor de Jóvenes del Fondo de Población de las Naciones Unidas (UNFPA), destacó la necesidad de que "los jóvenes adquirieran una información adecuada para conocer y entender su sexualidad, debido a que comienzan su vida activa con escasos conocimientos acerca del tema, lo cual ha provocado que cada año 14 millones de adolescentes de entre 15 y 19 años de edad den a luz, y que en países en desarrollo aproximadamente la mitad de las adolescentes ya son madres antes de cumplir 18 años, aun cuando el riesgo de muerte a esta edad es dos veces superior al de una mujer de entre 20 y 30 años" (Conapo, 2007, p. 3).

${ }^{2}$ Encargado del Despacho de la Secretaría General del Conapo.

${ }^{3}$ Según palabras del Mtro. Fernández Varela, Secretario General del Conapo. 
y radical en la impartición de la educación sexual con una perspectiva de género de las escuelas, haciendo énfasis en el escenario de la violencia juvenil en Baja California, México, aunque ésta es una situación que se inserta en un marco internacional.

Con este fin, a partir de una metodología de búsqueda documental y con el uso del método deductivo, se ofrece una panorámica que destaca la magnitud alcanzada por la violencia de pareja entre los jóvenes, sus preocupantes consecuencias y la dificultad cada vez mayor de su abordaje, debido a la normalización de tales conductas. Aspecto que a su vez obedece a la resistencia de implementar una adecuada educación sexual y de género. Se explicita la estrecha relación que existe entre ambas prácticas. Se ilustra con diversas investigaciones desde el punto de vista internacional y nacional. Y se hace hincapié en las cifras en las que se presenta la violencia en los jóvenes de Baja California, debido a que, a pesar de ser ésta una de las entidades de mayor crecimiento poblacional del país, este crecimiento se ubica en los últimos lugares en cuanto a su rezago educativo (Navarro, 2009), por lo que ya han sido destacados, entre otros, los altos valores que presenta en el embarazo adolescente (González y Vega, 2013, p. 150) debido a la falta de atención de la sexualidad como parte importante de su desarrollo (Ángel, 2012).

\section{Sobre la violencia de pareja. ${ }^{4}$ Situación internacional, nacional y estadísticas de Baja California}

McDonell, Ott y Mitchell (2010), Kaukinen, Gover y Hartman (2012), y Rojas (2013) concuerdan con los datos expresados por la Organización Mundial de la Salud (oms) (Jiménez, 2007, citado en Velázquez, 2011) respecto de que las agresiones en el noviazgo ya son frecuentes en al menos una tercera parte de la población explorada. En Estados Unidos, de donde proviene buena parte de la literatura sobre este tema, la prevalencia de adolescentes victimadas por su pareja oscila entre 18 y 32\% (Howard y Wang, 2003, citados en Rey-Anacona, 2009); aunque

${ }^{4}$ Por las características de las muestras que aparecen en los estudios analizados, consideramos importante aclarar que en todos los casos de este trabajo, las referencias pertenecen a parejas heterosexuales. 
en diferentes estudios (Molidor, 2005; Centers for Disease Control and Revention, 2006; Cornelius y Resseguie, 2007; Muñoz-Rivas, Graña, O'Leary y González, 2007; Sears, Biers y Price, 2007) se plantean cifras que rebasan $80 \%$ de ocurrencia entre ambos miembros. Otros autores comparten el hecho de que suelen ser agresiones mutuas (Glass et al., 2003; Dixon, Archer y Graham-Kevan, 2012; Karakurt y Cumbie, 2012; Kaukinen, Govern y Hartman, 2012). En España, Muñoz-Rivas y colaboradores (2007), al analizar la frecuencia de conductas verbales ofensivas en una muestra de varones y mujeres de entre 16 y 20 años de edad, señalaron que ésta alcanzó $95.3 \%$ en las mujeres y $92.8 \%$ en los varones.

En nuestros países, la Comisión Económica para América Latina y el Caribe (CEPAL) señalaba en 2008 el crecimiento de estos problemas en ambos sexos (CEPAL, 2009). La Encuesta Nacional de Victimización Criminal de Chile (Tolan, Gorman-Smith y Henry, 2006) expuso tasas de maltrato íntimo que aumentaron entre las féminas de 15 a 19 años, alcanzando su máxima expresión entre los 20 y los 24 años, por lo que la adultez temprana despuntó como el periodo de la vida con mayor riesgo de violencia. También en este país, Flores, Gajardo, Mardones y Uribe (2004) destacaron que las consecuencias más graves eran para la mujer, encontrando entre ellas una predominancia de la violencia psicológica, en lo que los hombres hacían uso principalmente de la violencia física. Y en Colombia, Rey-Anacona (2009) mostró que 82.6\% de adolescentes y jóvenes adultos informó acerca de algún acto de maltrato por parte de su pareja, sin diferencias significativas entre varones y mujeres victimizados $\mathrm{u}$ objeto de al menos una forma de maltrato (sobre todo verbal) por parte de su pareja. En este sentido, vale resaltar no sólo el incremento de esta violencia, sino su duplicidad (inconsciente por invisibilizada y aceptada por normalizada) en ambos sexos, lo cual podría sumarse al hecho de entender que las diferencias entre las construcciones identitarias tanto de hombres como de mujeres también implican relaciones de género-poder múltiples (Connell, 1996, p. 39; Rivero, 2010).

México continúa estos mismos patrones en tanto, como refleja Rojas (2013), en diversas investigaciones se recalca que la perpetración y la victimización suelen ser tan frecuentes en el noviazgo que al menos ocupan una tercera parte de la población joven estudiada. Y como estas prácticas, que comienzan con un desacuerdo, se tornan cada vez más crónicas, 
permitidas y legitimadas en la sociedad (Narváez, 2012, pp. 79-80), han alcanzado resultados tan alarmantes que muchas novias han llegado a cambiar su comportamiento, su forma de vestir e incluso su contacto con amigos, familiares y compañeros de escuela, con tal de evitar que su pareja se moleste (Vázquez, Grajeda y Domínguez, 2012, p. 619). La agresión psicológica es la que se presenta con mayor frecuencia, según varios estudios (Trujano, Nava, Tejeda y Gutiérrez, 2006; González, 2007; Martínez y Reyna, 2010; Cortaza, Mazadiego y Ruiz, 2011; Escoto, Arias y Amador, 2012; Trujillo, Anda y Montoya 2012), especialmente la de tipo verbal (Delabra, Ríos, Hernández y Villarreal, 2010; Estrada y Rodríguez, 2011; Rojas, 2013).

También de los datos derivados de la Encuesta Nacional de Violencia en las Relaciones de Noviazgo (ENvin) del Instituto Mexicano de la Juventud (Imjuve, 2007) se desprende que 76\% de las y los mexicanos con edades entre los 18 y los 24 años padeció agresiones psicológicas, 15\% experimentó al menos un incidente de violencia física, y $16.5 \%$ de las jóvenes mencionó haber sufrido un evento de violencia sexual. También es importante señalar que, según resultados del Imjuve-SEP (2007, p. 14), la mayor proporción de quienes recibieron violencia física fue del sexo femenino (61.4\%), aunque ambos sexos la llevaron a cabo (46\% de hombres la recibió a su vez). También declararon como las principales formas de violencia entre las parejas adolescentes: "la prohibición de relaciones de amistad, las burlas, el abuso sexual y las agresiones físicas como empujones o golpes" (Méndez, 2012), lo cual coincide, entre otros, con el estudio realizado por Moral de la Rubia López, Díaz y Cienfuegos (2011).

La titular de la Secretaría de la Juventud (sejuvec), Andrea Spinolo, dio a conocer recientemente que "en Querétaro, tres de cada 10 jóvenes sufren violencia durante el noviazgo y, de ellos, el $70 \%$ corresponde a la violencia psicológica" (Patiño, 2013). Menciona además que "este tipo de violencia es preocupante, porque incide directamente en la mentalidad de los jóvenes de 15 a 29 años". E incluso, como algo sustancial, destacó: "Lo que estamos midiendo es el tema de la información. ¿Qué tanto tiene información [el joven] al respecto?" (como se cita en Patiño, 2013).

Otro instrumento que ha dado cuenta de la violencia de pareja y de otro tipo de violencia contra la mujer, es la Encuesta Nacional sobre la 
Dinámica de las Relaciones en los Hogares (ENDIREH), realizada por el Instituto Nacional de Estadística y Geografía (INEGI) en los años 2003, 2006 y 2011. De aquí es importante apuntar que en 2006 la ENDIREH reportó a escala nacional a $46.7 \%$ de mujeres violentadas por su pareja a lo largo de la relación (INEGI, 2008, p. 7).

En este contexto nacional, Baja California, no obstante ser una de las entidades de mayor crecimiento poblacional de los 32 estados del país, presenta un notable estancamiento educativo ${ }^{5}$ y problemas derivados del ejercicio de una sexualidad poco informada entre los jóvenes (González y Vega, 2013, p. 150), con una alta cifra de embarazos adolescentes (Ángel, 2012), atraso que requiere de un gran esfuerzo en su sector educativo por parte del gobierno estatal y la sociedad civil (Navarro, 2009).

Desde el punto de vista social, esto se expresa en que, desde el año 2008, se reportó que por cada 100 mujeres, más de un tercio (34.6\%) había sido objeto de maltrato por parte de su pareja, sobre todo de tipo emocional. ${ }^{6}$ Evidenciando esto, que es en el ámbito doméstico (íntimo) donde se vive mayormente la violencia contra las mujeres y que su prevalencia por edad es más frecuente en las edades más jóvenes (INEGI, 2008).

Los resultados de la ENDiReH en 2011 demuestran que en México (o sea, a escala nacional) $44.9 \%$ de las mujeres mayores de 15 años había sufrido violencia por parte de su pareja; es decir, se registró un descenso en este rubro en relación con la encuesta anterior. Pero en lo que a Baja California se refiere, se señala a $38.6 \%$ entre las mujeres mayores de 15 años con la misma situación. Lo que indica un incremento de cuatro puntos porcentuales en el estado, entre 2008 y 2013 (INEGI, 2013, p. 9), ${ }^{7}$ como se aprecia en la figura 1.

${ }^{5}$ De acuerdo al estudio "Contra la pared. Estado de la Educación en México 2009", que además señala que "El gobierno estatal de Baja California y la sociedad civil deben pensar y trabajar en políticas públicas innovadoras, tanto educativas como de desarrollo social, que aseguren un sistema estatal de educación básica inclusivo, eficaz y de calidad" (Navarro, 2009).

${ }^{6}$ De las mujeres que reconocieron haber sufrido violencia por parte de su pareja, $83.1 \%$ había experimentado violencia emocional, 50.2\% violencia económica, 39.5\% violencia física y $14 \%$ violencia sexual (INEGI, 2008, p. 8).

${ }^{7}$ En 2011, de las mujeres que reconocieron haber padecido violencia por parte de su pareja, 91.5\% había sido de tipo emocional, $50.5 \%$ experimentó violencia económica y $21.1 \%$ y $10.4 \%$ soportó violencia física y sexual respectivamente (INEGI, 2013, p. 11). 
Figura 1. Mujeres mayores de 15 años que padecieron violencia por parte de su pareja en México y Baja California

(Comparativa ENDIREH 2006 y 2011)

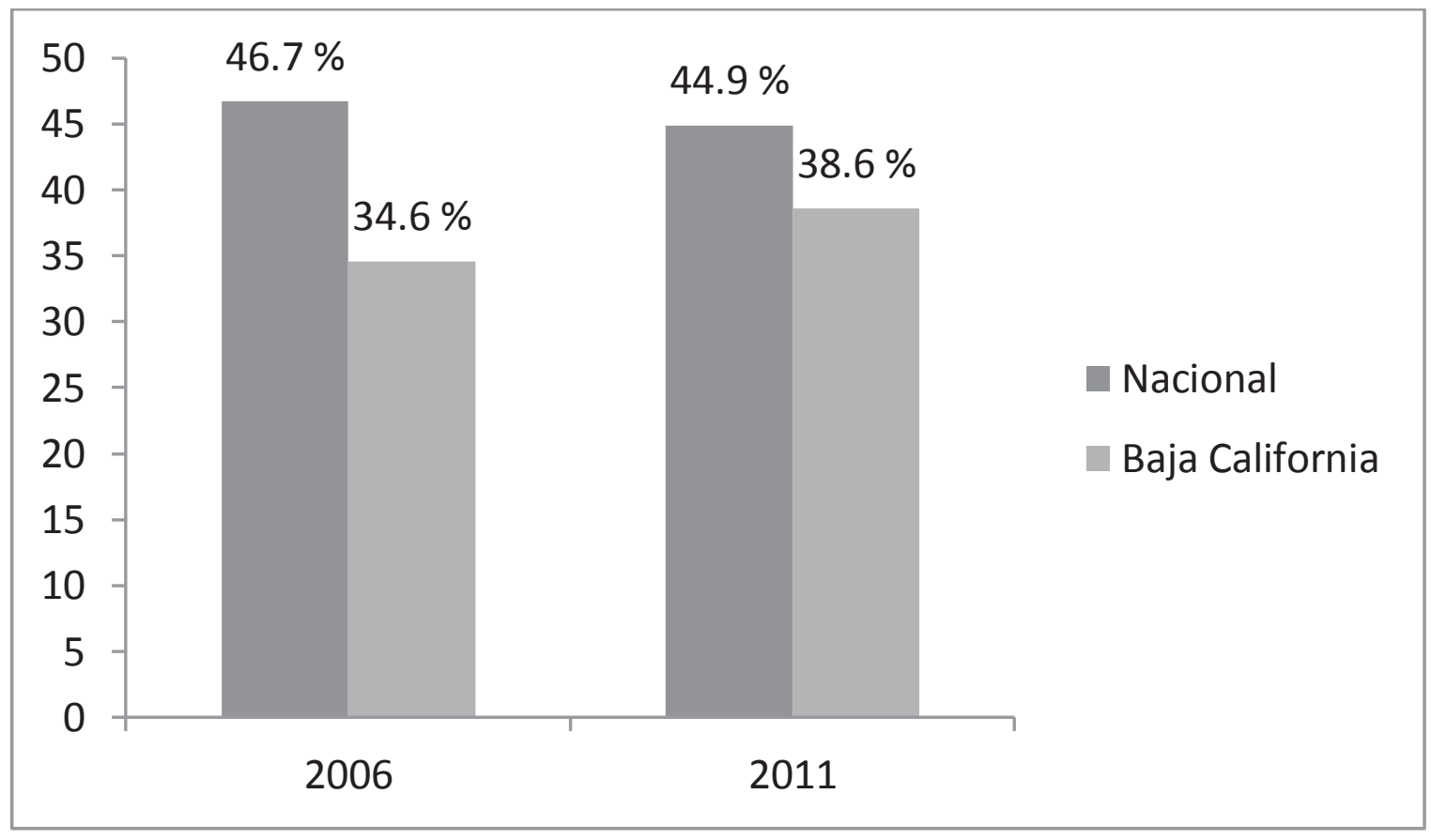

Fuente: Elaboración propia a partir de los resultados de la ENDIREH 2006 y 2011 (INEGI, 2008 y 2013 ).

Conjuntamente con la visibilidad y la existencia de la violencia contra las mujeres en el ámbito privado a escala nacional y de su incremento en el caso de Baja California en 2011, otro aspecto fundamental es que los jóvenes sigan siendo el sector de la población más vulnerable, en comparación con los adultos. Ya en 2007, según la Encuesta Nacional de Violencia en las Relaciones de Noviazgo (ENVINOv), la población con edades de entre 15 y 24 años registraba una incidencia de $38 \%$ en violencia sexual y $35 \%$ en violencia emocional (Imjuve, 2007). (Véase cuadro 1).

Por su parte, la ENDIREH (2006) señalaba que en México las mujeres jóvenes de 15 a 29 años constituían el grupo etario que más padecía violencia por parte de su pareja, con $46.2 \%$ en 2011. Grupo que permanece aún como el más vulnerable, con 38.4\% (INEGI, 2008 y 2013). (Véase figura 2). 
Cuadro 1. Jóvenes entre 15 y 24 años de edad que mantenían una relación de noviazgo (en los últimos 12 meses) sin vivir con su pareja en el mismo hogar que padecieron violencia

\begin{tabular}{|lcc|}
\hline & Hombre & Mujer \\
\hline 6.8\% sufrió violencia física & $10.3 \%$ & $2.9 \%$ \\
$39.4 \%$ sufrió violencia emocional & $42.7 \%$ & $35.8 \%$ \\
$8.2 \%$ violencia sexual & $46.1 \%$ & $38.4 \%$ \\
\hline
\end{tabular}

Fuente: Encuesta Nacional de Violencia en las Relaciones de Noviazgo 2007, Instituto Mexicano de la Juventud, 2007.

Figura 2. Mujeres que sufrieron violencia por parte de su pareja según grupos de edad en México (ENDIREH 2006 y 2011)

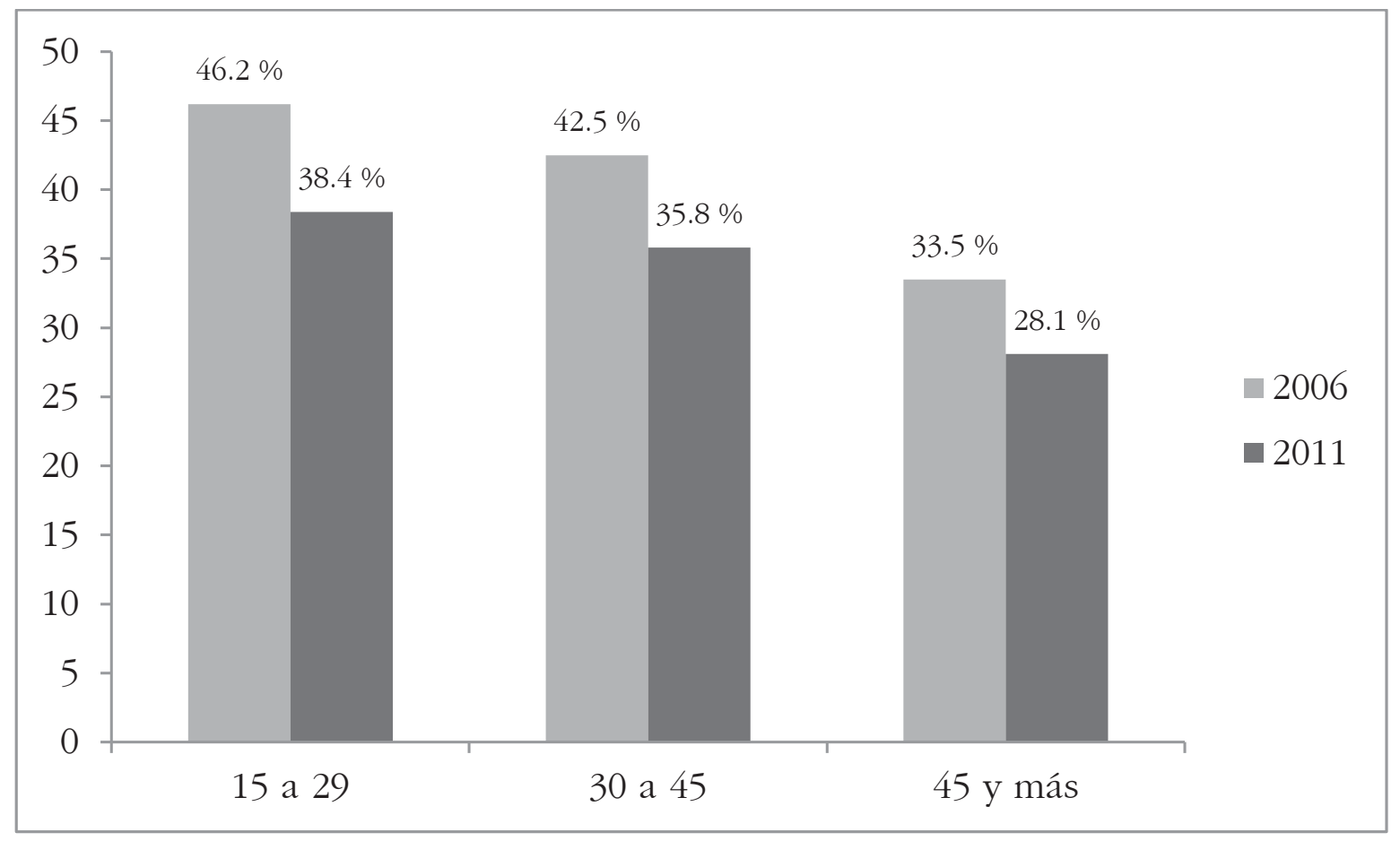

Fuente: Elaboración propia a partir de los resultados de la ENDIREH 2006 y 2011 (INEGI, 2008 y 2013). 
En el caso de Baja California, la situación es similar. La ENDIREH (2006) reportaba que las mujeres de 15 a 29 años eran las que más sufrían violencia por parte de su pareja, con 35.2\%. Dato que se confirma para 2011, con $34.4 \%$, frente a los otros grupos de edad (INEGI, 2008 y 2013) (véase figura 3).

Además, es de resaltar que la población de 15 a 24 años es la de mayor fragilidad ante la violencia emocional, ${ }^{8}$ aparte de su aumento significativo $^{9}$ (de casi tres puntos porcentuales) entre los que poseen de $15 \mathrm{a}$ 19 años en cuanto a la violencia física. Véase la figura 4 para estos fines, donde, por otro lado, es notorio el paulatino descenso de este maltrato en relación con la edad.

Figura 3. Mujeres que sufrieron violencia de parte de su pareja por grupo de edad en Baja California (ENDIREH 2006 y 2011)

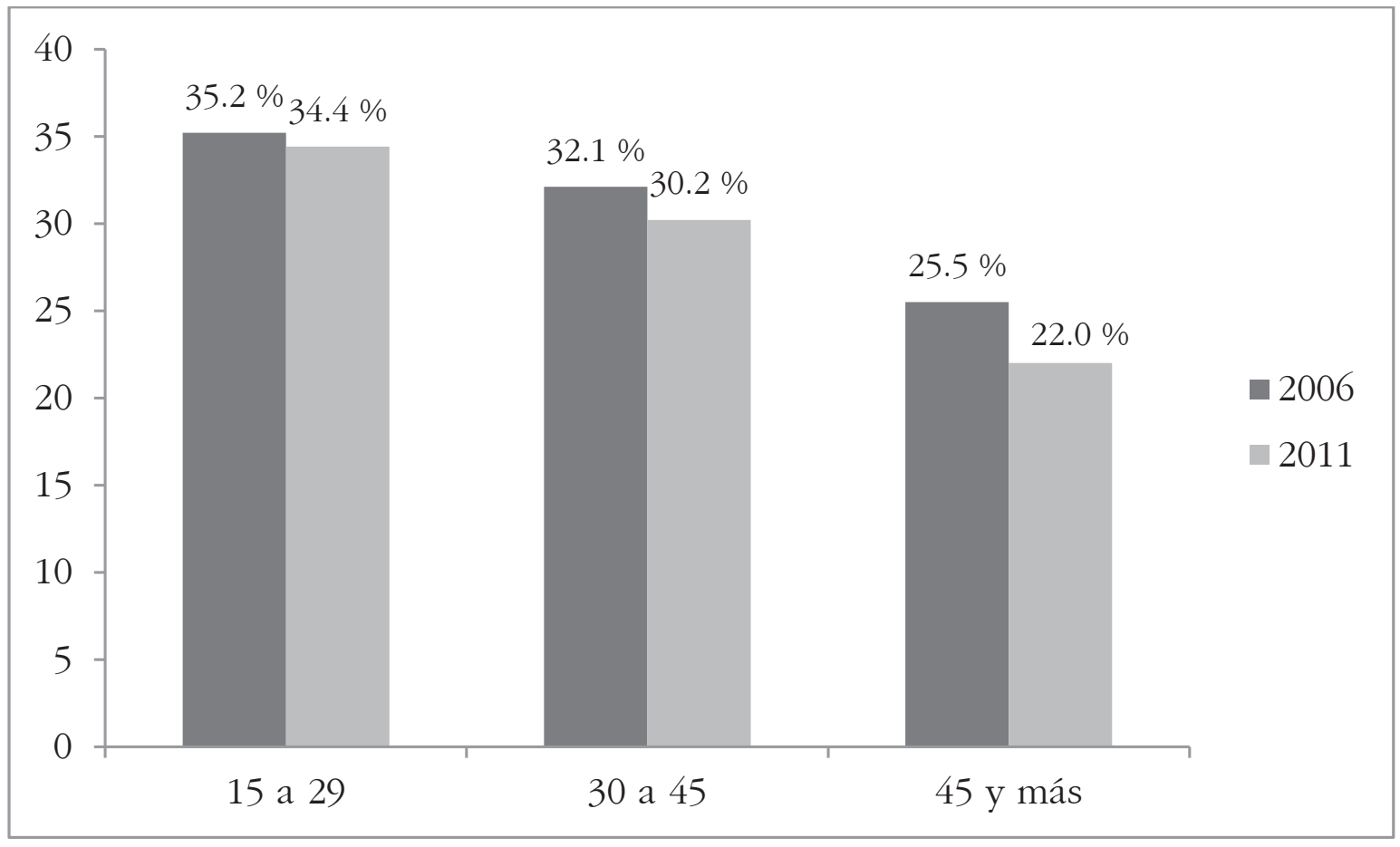

Fuente: Elaboración propia a partir de los resultados de la ENDIREH 2006 y 2011 (INEGI, 2008 y 2013).

${ }^{8}$ Con un porcentaje de incidencia de aproximadamente $31 \%$.

${ }^{9}$ Con respecto a la ENDiReH 2003. 


\section{Figura 4. Mujeres que padecieron violencia emocional por grupo de edad}

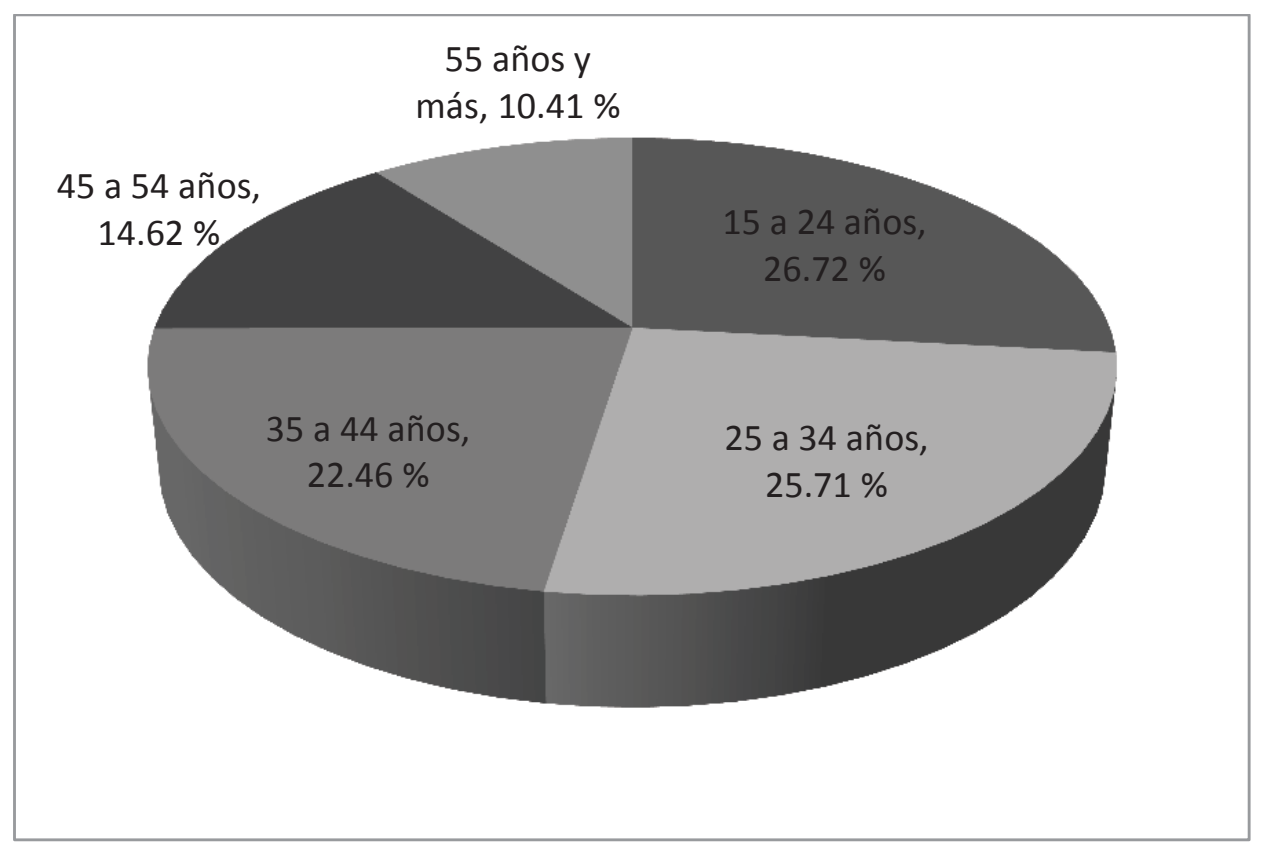

Fuente: Elaboración propia a partir de los resultados preliminares de la ENDIREH 2011 en su versión tabulada disponible en el INEGI (2011).

Incluso, en la totalidad de las mujeres que padecieron violencia por parte de su pareja en el estado bajacaliforniano, la de tipo psicológico (llamada de carácter emocional) coexiste con mayor presencia que el resto (la económica, la física y la de tipo sexual) (ver figura 5).

Es por lo tanto un hecho palpable que los jóvenes constituyen el sector de la sociedad que más experimenta situaciones de violencia en el ámbito privado, siendo además visible que a mayor edad, empieza a disminuir el padecimiento de violencia hacia las mujeres por parte de su pareja. En tal sentido, remitiéndonos a los últimos resultados de la ENDIREH de 2011 - realizada por el INEGI en conjunto con el Instituto Nacional de las Mujeres (INM) - , vemos que, en relación con la ENDIREH de 2006, también se destaca que son los jóvenes (entre 15 y 24 años) los que se mantienen como los más vulnerables, en comparación con el resto de la población.

Por otro lado, indagando sobre el sentir y pensar frente a ciertos roles socialmente esperados - en un acercamiento a las raíces culturales de esta problemática一, se encontró que (en pleno siglo xxı, año 2011) de las 
Figura 5. Condición de violencia en la pareja en Baja California

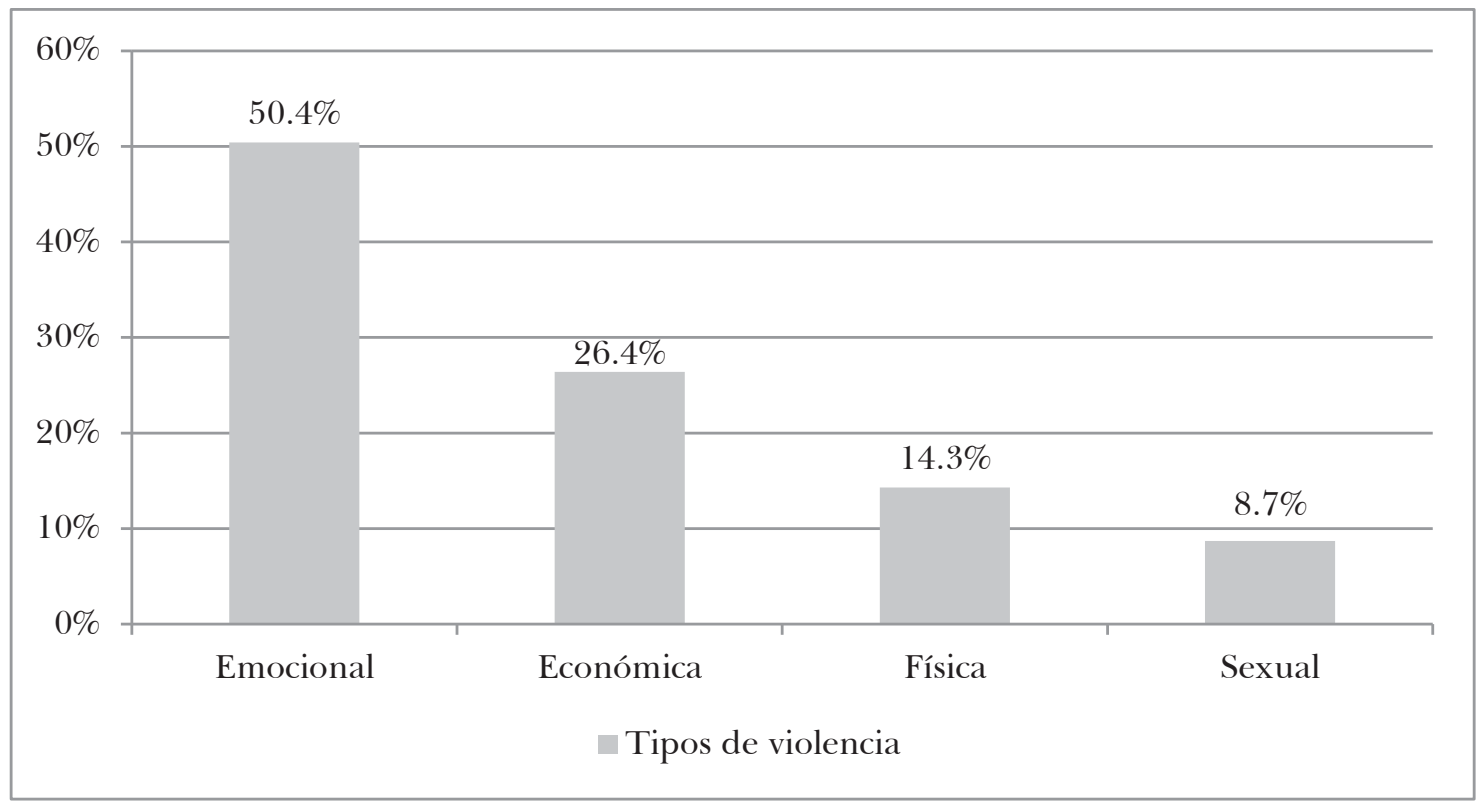

Fuente: Elaboración propia a partir de resultados de la ENDIREH 2011 en su versión tabulada disponible en el INEGi (2011).

* Mujeres de 15 años y más según tipo de violencia hacia ellas a lo largo de la relación con su última pareja.

mujeres que sufrieron violencia por parte de su pareja (a partir de los 15 años), casi un tercio manifestó estar de acuerdo en que si hay golpes o maltrato en su casa es un asunto interno, o sea, de familia, y que por lo tanto no debe divulgarse: ahí debe quedar. Y aproximadamente un séptimo de esta población ( $16.8 \%$ y $14.7 \%$, respectivamente) estuvo de acuerdo en que "una esposa debe obedecer a su esposo o pareja en todo lo que él ordene", así como que "es obligación de la mujer tener relaciones sexuales con su esposo o pareja" (ENDIREH, 2011). Con estos preceptos, que continúan evidentemente trasmitiéndose de generación en generación, se aprecia la ardua labor que aún existe por delante.

El estudio de González y Fernández (2010) sobre género y maltrato de pareja en jóvenes de Baja California evidencia cómo, de una muestra compuesta por cuatro mil personas de 18 a 29 años, una parte significativa coexistió en un ambiente de violencia familiar y era dada a repetir esta 
conducta en sus actuales relaciones de pareja, sea en el papel de víctima o en el de victimario. Incluso, aquellos que no reconocían haber sido perjudicados con estas situaciones en su familia de origen, describían como "naturales" las conductas violentas. Las percibieron tan "normales", que algunos castigos infringidos, corporales o de otro tipo, fueron denominados como "merecidos" (véase figura 6).

Esta "normalidad" de la violencia se ve también reflejada en los datos arrojados por la ENVINOv de 2007 realizada por el Imjuve, donde 62.2\% de las mujeres, e incluso $51.8 \%$ de hombres, que sufrieron violencia física por parte de su pareja, no recurrió a nadie, ya que consideraron que "no tenía importancia"; $9.5 \%$ de ellas y $16.8 \%$ de ellos pensaron que "eso es algo normal en una relación de noviazgo". Y, más preocupante aún: al hablar de violencia sexual, $46 \%$ de las jóvenes entrevistadas entendió que esta agresión "no tenía importancia” (véase cuadro 2).

Como plantean Ramírez y Núñez (2010), existe el peligro de que "vivir con violencia se convierta a la postre en una forma de vida erróneamente natural y que las personas pueden acostumbrarse a ella sin percatarse de que los episodios violentos ocurran con más frecuencia y mayor intensidad" (p. 275).

Figura 6. Causas de violencia contra la mujer

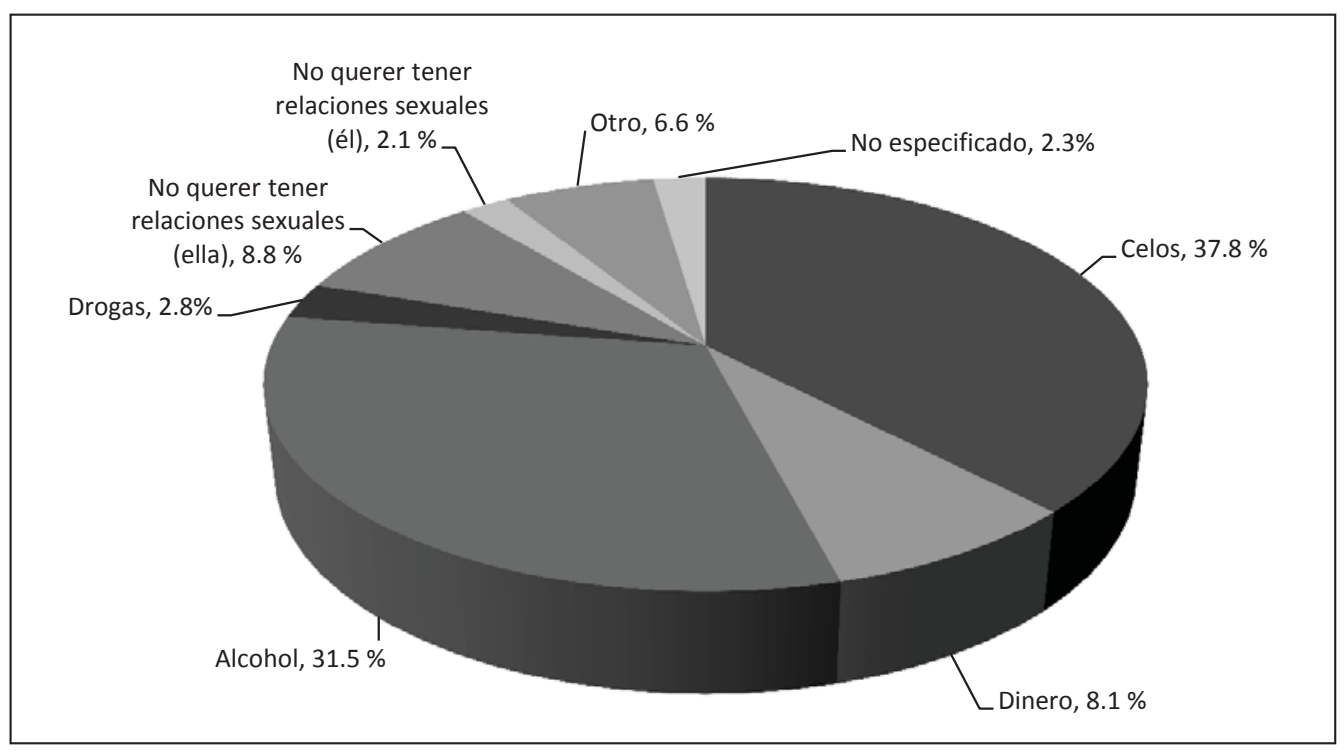

Fuente: González y Fernández (2010, p. 120). 
Cuadro 2. Razones por las que las y los jóvenes de 15 a 24 años no recurren a nadie en caso de sufrir violencia

\begin{tabular}{|lcc|}
\hline \multicolumn{1}{|c|}{ Violencia física } & Hombre & Mujer \\
\hline "No tiene importancia" & $62.2 \%$ & $51.8 \%$ \\
"Es normal en una relación de noviazgo" & $16.8 \%$ & $9.5 \%$ \\
\hline \multicolumn{1}{|c|}{ Violencia Sexual } & Hombre* & Mujer \\
\hline "No tiene importancia" & & $46 \%$ \\
"Es normal en una relación de noviazgo" & & $4.6 \%$ \\
"Le da vergüenza" & & $24.1 \%$ \\
\hline
\end{tabular}

Fuente: Encuesta Nacional sobre Violencia en el Noviazgo 2007, Instituto Mexicano de la Juventud, 2007.

* Sólo se preguntó a las mujeres en los casos de violencia sexual.

\section{La educación sexual con perspectiva de género en las escuelas. Concepto, situación e importancia}

La sexualidad humana involucra mucho más allá de la genitalidad y de la reproducción. Es "una construcción social relacionada con las múltiples e intrincadas maneras en que nuestras emociones, deseos y relaciones se expresan en la sociedad en que vivimos" (Montero, 2011, p. 250), donde "los aspectos biológicos condicionan en parte esta sexualidad, proporcionando la fisiología y morfología del cuerpo las condiciones previas para esta construcción” (Dides, Benavente y Morán, 2009, p. 8). El concepto de salud sexual, como bien enfatiza Montero, se relaciona con la integración de los aspectos somáticos, emocionales, intelectuales, sociales y culturales del ser sexual. Enriquece y fortalece la personalidad y el amor, vinculándose:

[...] con la capacidad que tenemos mujeres y hombres de disfrutar y expresar nuestra sexualidad, sin coerción, violencia ni discriminación y sin riesgo de adquirir infecciones transmitidas sexualmente ni de tener embarazos no planificados o no deseados. Por ende, está encaminada al desarrollo de la vida y de las relaciones personales (Montero, 2011, p. 1251). 
Significa entonces poder sentir y expresar el placer y disfrutar de las relaciones sexuales deseadas y responsablemente escogidas, sin que impliquen un riesgo psíquico ni físico para la salud. Así como tener acceso a la educación sobre estos tópicos y a una atención integral, que incluya el derecho de acceder de forma amplia y actualizada a la información sobre el tema, admisión a los métodos de regulación de la fertilidad y a todos los servicios de salud para ambos sexos (oms, 1995; Montero, 2011). En este sentido, como expresan García, Cañadas, González, Fernández y García (2011), si bien el comportamiento sexual en la edad adulta se debe caracterizar por los principios éticos y universales de tolerancia, respeto y ausencia de imposiciones y discriminaciones, en la población adolescente "éstos deben cumplirse aun más, puesto que [los adolescentes] carecen de los conocimientos y de la experiencia necesarios para afrontar situaciones no deseables" (López, 2005; Guerrero, M. D., Guerrero M., García y Moreno, 2008, p. 52) y existe el riesgo de tomar decisiones inadecuadas respecto de las responsabilidades inherentes a la sexualidad, sea por falta de información, por una inadecuada influencia social o por la incapacidad de poder prever y planificar las consecuencias futuras en ese momento (Guerrero et al., 2008).

Este tipo de circunstancias puede desembocar en embarazos adolescentes no deseados, infecciones de transmisión sexual (ITS) y altas tasas de VIH/sida (Martini, 2007; Kang, Skinner y Usherwood, 2010; Rodríguez, Sanabria, Contreras y Perdomo, 2013), que en México alcanzan actualmente 106.2 casos por cada 100000 habitantes, siendo que la mayor parte $(78.6 \%$ de los casos) se encuentra comprendida precisamente en el grupo de edad de 15 a 44 años. Y que entre ellos, Baja California es la segunda entidad con más infecciones de sida ${ }^{10}$ — después del Distrito Federal-, con 169.6 casos por cada 100000 habitantes (Saavedra y Bravo, 2009, p. 22). Además, según un estudio realizado en mujeres embarazadas del Hospital General de Tijuana, se encontró que éstas poseían

${ }^{10}$ Aspecto ratificado posteriormente por González y Vega (2013) al analizar las problemáticas del comportamiento sexual y reproductivo de las jóvenes inmigrantes de Baja California, pues encontraron que éstas presentaban la mayor tasa de enfermedades de transmisión sexual y los más altos niveles de embarazos no deseados, riesgo que pudiera haber sido evitado, en gran medida, si el país dispusiera de una educación sexual oportuna, de calidad y de amplia cobertura. 
"una prevalencia de viH/sida de $1 \%$, mientras que la estimada en la población general es de $0.3 \%$. Siendo además que la infección por el viH se asoció con tener un mayor número de parejas sexuales y el no haber recibido atención prenatal, entre otras causas" (Kendall, 2009, pp. 152-153). ${ }^{11}$

De igual manera, una impartición adecuada de educación sexual propicia el respeto a la diferencia y la necesaria desaparición de los papeles de género estereotipados;

en este caso, de los hombres y mujeres mexicanos, que marcados por una notoria desigualdad, afectan tanto la aparición de serios problemas en el área sexual como las condiciones inequitativas en la pareja, afectando principalmente a la mujer y contradiciendo la esencia de lo que idealmente debería ser una estructura armónica y cooperativa (Álvarez-Gayou, como se citó en Serrano, 2012).

$\mathrm{Al}$ ser parte obligatoria de la instrucción integral del alumno/a, lo que esta instrucción promueve es una personalidad sana y una sexualidad responsable, vinculada al amor, a la solidaridad y a la equidad de género, bajo la forma de la dignidad del hombre y de la mujer. Como bien señala Treviño (2012, p. 93),

debemos cambiar la educación desde el aula, para no seguir repitiendo los roles de género que hacen daño a las niñas y a los niños [y para esto], debemos cambiar la cultura, mujeres y hombres, a nivel personal: desaprendiendo todo lo que nos enseñaron que es nuestro rol de género.

Y, de acuerdo con Martínez (2012):

Las significaciones e interpretaciones de la violencia parten de una directriz de género que atraviesa y condiciona todos los territorios de la vida cotidiana, [por lo que educar bajo esta vertiente] permite entender con mayor profundidad las significaciones que elaboran los sujetos, partiendo de su identidad de género-corporal-sexual y de su sentir-estar como hombres o como mujeres jóvenes en un entorno determinado (p. 173).

${ }^{11}$ Y el tener una pareja usuaria de drogas inyectadas. 
Pero esta implementación — tan relacionada con las expectativas socioculturales que se tienen acerca del papel a seguir, en dependencia de cómo se interpreten las diferencias biológicas sexuales- atraviesa el campo educativo mexicano plagado de ausencias, distorsiones y diferencias a lo largo del país (González, 2009). Y siendo que, "por regla general, los regímenes de género de las escuelas refuerzan la dicotomía de género", si no tratamos de establecer la justicia de género en las escuelas, lo que se ofrecerá a los chicos será una educación degradada (cf. Connell, 1996, p. 1). Ratificándose que, como son los profesores los que constituyen la mano de obra de la reforma educativa, para que ocurra algo importante en las escuelas, son ellos ante todo los que han de empeñarse en que esto se produzca.

Sin embargo, como apunta Caricote (2008), tanto la mayoría de los docentes como los propios progenitores están muy marcados por una historia de represión sexual, por lo que albergan y trasmiten sentimientos tan confusos y negativos, que lo que logran es confundir y censurar la indagación de los adolescentes. O, como parte de esta misma actitud prejuiciosa, enfocan la información únicamente en la genitalidad, sin tomar en cuenta la integralidad del adolescente como individuo, con necesidades tanto físicas como psicológicas y socioculturales, que se expresan y forman parte activa de esta área. Por ello es que para que los maestros estén en condiciones de obrar como educadores y orientadores sexuales verdaderos y eficientes, deben primero estar listos para crecer, controlar y superar sus deficiencias, limitaciones y contradicciones (Lescaille, Odelín, González, Alfonso y Arencibia, 2009). ${ }^{12}$

Esto conlleva a un largo e intrincado proceso de desarticulación, dado que, desde el principio de la década de 1950, cuando "el Estado mexicano y la jerarquía católica aunaron criterios en cuanto a lo que sería considerado 'decente' o 'inmoral', sobre todo en lo concerniente a la juventud" (Pérez, 2011, p. 380), la negación de la sexualidad en el país se ha perpetuado hasta el presente siglo. Y ya no sólo derivada de estas influencias, sino que además, esta visión, tan imbricada en la cultura, aún sigue siendo vista como "natural", "la única opción moral" y la "única verdad" (Collignon, 2011); por lo que se concibe a la sexualidad como sinónimo

${ }^{12} \mathrm{Y}$ en esto ellas se refieren a los orientadores en este tema en América Latina en general. 
del "sexo-pecado", de la "negación del placer" y con un elevado nivel de culpabilidad (Mejía, 2003, pp. 46-47). Según Climent (2009):

Resistencias al cambio de los sectores más conservadores de la sociedad que aún persisten, a pesar de los avances en la legislación sobre salud y derechos sexuales y reproductivos [se deben] al peso de la ideología e idiosincrasia imperantes entre quienes diseñan las políticas públicas y toman las decisiones [para las escuelas y la sociedad en su conjunto; al punto de que hay] autoridades que han impedido incorporar la discusión sobre sexualidad, género y derechos sexuales y reproductivos en centros escolares (incluso universitarios), bajo la excusa de que ello "politiza" a los alumnos (pp. 236-275).

Esto (que no es patognomónico de México), ha llegado al grado de que sean estigmatizados algunos docentes que se han atrevido a sugerir este tipo de temáticas, según plantean Lescaille y colaboradores (2009).

\section{Conclusiones}

A partir de lo anteriormente planteado, insistimos en que, en el marco de la desnaturalización de la violencia, sobre todo entre las parejas jóvenes, se priorice el trabajo preventivo, incorporando por igual a ambos sexos. Donde también se evidencie que la identidad masculina no requiere de un modelo de maltrato y de negación de sus emociones para una interacción equitativa con la pareja y con el entorno en el que se desarrolla.

Ello implica contar con la impartición de una educación sexual responsable en las escuelas ${ }^{13}$ que influya en la formación integral del alumno y desarrolle actitudes favorables hacia la igualdad, la equidad y la diversidad (Galván et al., 2012, p. 202), y también la elaboración de instrumentos de evaluación, para medir los resultados de su implementación, ${ }^{14}$ lo que viabiliza la retroalimentación necesaria que valide el impacto ejercido. ${ }^{15}$

${ }^{13}$ De la que, al menos en el currículum de los planteles del estado de Baja California, y a pesar de su extrema importancia, se carece por completo.

${ }^{14}$ Que están enfocados en un alto porcentaje solamente a mujeres víctimas de violencia y la atención a éstas. Véase Valdez-Santiago, Torres, Garda, Gómez y Quiroz (2008).

${ }^{15} \mathrm{Al}$ respecto, la autora desea mencionar un proyecto piloto instrumentado en 
Tarea mancomunada que obliga a una apertura entre las instancias generadoras de políticas públicas (INEGI, 2013, p. 299), las instituciones sociales y científicas y la población en general.

En esta misma línea, es importante que la categoría de género se entienda no como sinónimo de mujeres, sino dentro de un plano de relación, donde la sociedad le otorgue igual valor, libertades, deberes y derechos a uno y a otro. Porque la mejor manera de luchar contra la discriminación nacida de la diferencia y de la disimetría entre los sexos, no es ni negarla ni eliminarla. Debemos extender la conciencia en ambos sexos para que estas campañas resulten efectivas, conscientes de que, como culmina Antoinette Fouque en sus Ensayos de feminología: "Dos no es el doble, sino el contrario de uno, de su soledad. Dos es alianza, doble hilo que no se puede romper" (De Luca, 2005, citado en Fouque, 2008, p. 30).

\section{Bibliografía}

Ángel, D. (7 de septiembre de 2012). Baja California: Falta educación sexual en adolescentes: El Colef. El Sol de Tijuana. Recuperado de < http://www.oem. com.mx/elsoldetijuana/notas/n2686188.htm >.

Caricote, E. (2008). Los docentes y la educación sexual en la adolescencia. Revista Ciencias de la Educación, 18(32), 13-33.

Centers for Disease Control and Prevention. (2006). Physical dating violence among High School students, United States, 2003. Morbidity and Mortality Weekly Report, 55(19), 532-535.

Comisión Económica para América Latina y el Caribe (CEPAL) (2009). Panorama social de América Latina 2008. Santiago de Chile: Comisión Económica para América Latina y el Caribe, Organización de las Naciones Unidas.

Climent, G. I. (2009). Entre la represión y los derechos sexuales y reproductivos: Socialización de género y enfoques de educación sexual de adolescentes que se embarazaron. La Ventana, 3(29), 236-275.

Collignon, M. M. (2011). Discursos sociales sobre la sexualidad: Narrativas sobre la diversidad sexual y prácticas de resistencia. Comunicación y Sociedad, (16), 133-160.

Ensenada, Baja California, donde se impartieron módulos de género y violencia entre jóvenes, y en cuya retroalimentación resultó que éstos ya habían pasado previamente por un módulo con objetivos similares pero del cual no captaron muchos conocimientos ni se produjo cambio alguno en sus actitudes al respecto (cf. Fernández, 2013). 
Consejo Nacional de Población (CONAPO). (27 de julio de 2007). Los jóvenes, alta prioridad para la política de población: Consejo Nacional de Población [Comunicado de prensa]. Recuperado de <http://www.conapo.gob.mx/es/CONAPo/Mexico_ DF_Viernes_10_de_agosto_de_2007_2>.

Consejo Nacional de Población (CONAPO). (2010). La situación actual de los jóvenes en México. México: Autor.

Connell, R. W. (1996). Teaching the boys: New reserch on masculinity, and gender strategies for schools. Teachers College Record, 98(2), 206-235.

Cornelius, T. y Resseguie, N. (2007). Primary and secondary prevention programs for dating violence: A review of the literature. Aggression and Violent Behavior, 12(3), 364-375.

Cortaza, L., Mazadiego, T. y Ruiz, S. (2011). Prevalencia de violencia en el noviazgo en estudiantes preuniversitarias de Minatitlán, México. Revista Exploratoris, 2, 13-18.

De Grazia, E. (1992). Girls lean back everywhere: The Law of Obscenity and the assault on genius. Nueva York: Random House.

Delabra, M., Ríos, E., Hernández, D. y Villarreal, M. (2010). Violencia de pareja y rendimiento escolar en estudiantes de bachillerato. Memoria Académica del I Congreso Latinoamericano de Ciencias de la Educación. Perspectivas hacia la Construcción de los Diálogos para una Sociedad Educadora, Mexicali, Baja California: Universidad Autónoma de Baja California.

Dides, C., Benavente, C. y Morán, J. (2009). Estado de la vinculación de la salud sexual y reproductiva, vIH y Sida e ITS en Chile. Santiago de Chile: Facultad Latinoamericana de Ciencias Sociales-Chile, Organización Panamericana de la Salud.

Dixon, L., Archer, J. y Graham-Kevan, N. N. (2012). Perpetrator programmes for partner violence: Are they based on ideology or evidence? Legal and Criminological Psychology, 17(2), 196-215.

Escoto, Y., González, M., Muñoz, A. y Salomon, Y. (2007). Violencia en el noviazgo adolescente. Revista Internacional de Psicología, 8(2), 1-33.

Estrada, C. y Rodríguez, F. (2011). Violencia y temor por la vida en mujeres jaliscienses violentadas por su pareja íntima. Revista Cubana de Salud Pública, $37(4), 462-471$.

Fernández, T. (2013). Taller de Integración Social y Autoestima. Estructura y resultados generales. Reporte final, El Colegio de la Frontera Norte. Recuperado de $<$ http://seminariovallesvitivinicolas.blogspot.mx/2013/01/view-reporte-finaltaller-autoestima-on.html>.

Flores, S., Gajardo, R. R., Mardones, G. y Uribe, L. (2004). Jóvenes universitarias que legitiman la violencia en sus relaciones de pololeo (Tesis), Universidad Católica de Temuco, Temuco, Chile.

Fouque, A. (2008). Hay dos sexos. Ensayos de feminología. México: Siglo xxi. 
Galván, L., Roldán, F., Castillo, C., Leyva, A., Obeso, A. y Alvidres, A. (2012). La mujer y la equidad de género en la educación superior: Caso ITSON. En L. Niño, L. Ovalle, R. Balbuena e I. González (Coords.). Los estudios de género en las fronteras de la violencia: Enfoques teóricos y metodológicos, reflexiones, experiencias y políticas públicas (pp. 190-204). Mexicali, Baja California: Universidad Autónoma de Baja California.

García, M., Cañadas, G., González, E., Fernández, R. y García, I. (2011). Educar en conductas sexuales saludables: Una innovación docente en promoción de la salud. Revista Médica de Chile, 139(10), 1269-1275.

Glass, N., Freland, N., Campbell, J., Yonas, M., Sharp, P. y Kub, J. (2003). Adolescent dating violence: Prevalence, risk factors, health out-comes, and implications for clinical practice. Journal of Obstet Gynecol Neonatal Nurs, 32(2), 227-238.

González, H. y Vega, G. (2013). Problemáticas del comportamiento sexual y reproductivo de las jóvenes inmigrantes de Baja California. Frontera Norte, 25(50), 131-154.

González, R. M. (2009). Estudios de género en educación: Una rápida mirada. Revista Mexicana de Investigación Educativa, 14(42), 681-699.

González, H. y Fernández, T. (2010). Género y maltrato: Violencia de pareja en los jóvenes de Baja California. Estudios Fronterizos, 11(22), 97-128.

González, S. (2007). Violencia en el noviazgo: Un estudio exploratorio entre estudiantes universitarias. Tesis de maestría. Universidad Autónoma de Nuevo León. Monterrey, Nuevo León.

Guerrero, M. D., Guerrero, M., García, E. y Moreno, A. (2008). Conocimiento de los adolescentes sobre salud sexual en tres institutos de educación secundaria valencianos. Revista Pediatría de Atención Primaria, 10(39), 49-58.

Instituto Nacional de Estadística y Geografía (INEGI). (2008). Panorama de violencia contra las mujeres. ENDIREH 2006. Baja California. Aguascalientes, México: Autor.

Instituto Nacional de Estadística y Geografía (INEGI). (2013). Panorama de violencia contra las mujeres en Baja California. ENDIREH 2011. Aguascalientes, México: INEGI, Instituto de la Mujer del Estado de Baja California.

Instituto Mexicano de la Juventud (Imjuve). (2007). Encuesta Nacional sobre Violencia en las relaciones de Noviazgo 2007. Recuperado de < http://www.inegi.org.mx/ est/contenidos/proyectos/encuestas/hogares/especiales/envin/default.aspx $>$.

Juárez, F. y Gayet, C. (2005). Salud sexual y reproductiva de los adolescentes en México: Un nuevo marco de análisis para la evaluación y diseño de políticas. Papeles de Población, (45), 177-219.

Kang M., Skinner, R. y Usherwood, T. (2010). Interventions for young people in Australia to reduce HIV and sexually transmissible infections: A systematic review. Sex Health, 7(2), 107-128. 
Karakurt, G. y Cumbie, T. (2012). The relationship between egalitarianism, dominance and violence in intimate relationships. Journal of Family Violence, 27(2), 115-122.

Kaukinen, C., Gover, A. y Hartman, J. (2012). College women's experiences of dating violence in casual and exclusive relationships. American Journal of Criminal Justice, 37(2), 146-162.

Kendall, T. (2009). Mujeres y vıн/sida. Una introducción a la vulnerabilidad femenina ante el virus. En VIH/sida y salud pública. Manual para personal de salud (pp. 145-154). México: Censida, Instituto Nacional de Salud Pública.

Lescaille, B., Odelín, D., González, M., Alfonso, K. y Arencibia, A. (2009). Valoración de la sexualidad en la adolescencia a partir de los estereotipos sexuales. MEDISAN, 13(2). Recuperado de < http://bvs.sld.cu/revistas/san/ vol13_2_09/san20209.htm>.

López, F. (2005). La educación sexual. Madrid: Biblioteca Nueva.

Martínez, H. y Reyna, Y. (2010). Violencia en el noviazgo (Tesis), Universidad Veracruzana, Minatitlán, Veracruz.

Martínez, C. (2012). Género, juventud y violencia. Significaciones a partir de la diversidad juvenil potosina. En L. Niño, L. Ovalle, R. Balbuena e I. González (Coords.), Los estudios de género en las fronteras de la violencia: Enfoques teóricos y metodológicos, reflexiones, experiencias y políticas públicas (pp. 159-175). Mexicali, Baja California: Universidad Autónoma de Baja California.

Martini, J. (2007). La educación sexual en tiempos del vin/sida. Enfermería Global, $6(2), 1-18$.

McDonell, J., Ott, J. y Mitchell, M. (2010). Predicting dating violence victimization and perpetration among middle and high school students in a rural southern community. Children and Youth Services Review, 32(10), 1458-1463.

Mejía, M. (2003). Sexualidad y derechos sexuales: El discurso de la Iglesia católica. Debate Feminista, 27, 45-56.

Méndez, C. (1 de septiembre de 2012). Jóvenes sufren violencia en el noviazgo en un 30\%: Imjuve. Milenio. Recuperado de < http://noticierostelevisa.esmas.com/ nacional/493709/sufren-30-jovenes-violencia-durante-noviazgo-imjuve/>.

Molidor, C. (2005). Gender differences of psychological abuse in high school dating relationships. Child and Adolescent Social Work, 12(2), 119-134.

Montero, A. (2011). Educación sexual: Un pilar fundamental en la sexualidad de la adolescencia. Revista médica de Chile, 139(10), 1249-1252.

Moral de la Rubia, J., Fuensanta L., Díaz, R. y Cienfuegos, Y. (2011). Diferencias de género en afrontamiento y violencia en la pareja. Revista Ces Psicología, $4(2), 29-46$.

Muñoz-Rivas, M., Graña, J., O’Leary, K. y González, P. (2007). Aggression in adolescent dating relationships: Prevalence, justification, and health consequences. Journal of Adolescent Health, 40(4), 298-304. 
Narváez, Z. (2012). Transformación y comprensión de las dinámicas de violencia, poder y las creencias de género en las relaciones de pareja mediante la intervención sistémica, (Tesis de maestría en Psicología Clínica), Pontificia Universidad Javeriana, Bogotá, Colombia.

Navarro, F. (27 de noviembre al 3 de diciembre de 2009). Exponen grave rezago educativo. Semanario Zeta. Recuperado de <http://www.zetatijuana.com/html/ Edicion1861/Reportajez_Exponen_grave_rezago_educativo.html>.

Organización Mundial de la Salud (OMS). (1995). Womens health. IV World Conference on Women. Beijing, China: Autor.

Patiño, A. (12 de febrero de 2013). Violencia psicológica en $70 \%$ de los novios queretanos. El Economista. Recuperado de <http://eleconomista.com.mx/ estados/2013/02/12/violenciapsicologica-70-novios-queretanos $>$.

Pérez, L. (2011). Censura y control. La Campaña Nacional de Moralización en los años cincuenta. Historia y Grafía, (37), 79-113.

Ramírez, C. y Núñez, D. (2010). Violencia en la relación de noviazgo en jóvenes universitarios: Un estudio exploratorio. Enseñanza e Investigación en Psicología, 5(2), 273- 283.

Rey-Anacona, C. (2009). Maltrato de tipo físico, psicológico, emocional, sexual y económico en el noviazgo: Un estudio exploratorio. Acta Colombiana de Psicología, 12(2), 27-36. Recuperado de < http://portalweb.ucatolica.edu.co/ easyWeb2/files/23_2732_v12n2-art2.pdf $>$.

Rivero, R. (2010). Las masculinidades en Cuba: Estudio con enfoque de diversidad e integración social. Sexología y Sociedad, 16(43). Recuperado de <http:// www.cenesex.sld.cu/webs/masculinidad.htm $>$.

Rodríguez, A., Sanabria, G., Contreras, M. E. y Perdomo, B. (2013). Estrategias educativas sobre promoción en salud sexual y reproductiva para adolescentes y jóvenes universitarios de Cuba. Revista Cubana de Salud Pública, 39(1), 161-174.

Rojas, J. (2013). Violencia en el noviazgo y sociedad mexicana posmoderna. Algunos apuntes sobre la figura del agresor y las agresiones bidireccionales. Uaricha, 10(22), 1-19.

Rubin, G. (1989). Reflexionando sobre el sexo: Notas para una teoría radical de la sexualidad. En C. Vance (Comp.), Placer y peligro. Explorando la sexualidad femenina (pp. 113-190). Madrid: Revolución.

Saavedra, J. y Bravo, E. (2009). Panorama del vin/sida en el 2006. viH/sida y salud pública. Manual para personal de salud (pp. 3-14). México: Censida, Instituto Nacional de Salud Pública.

Sears, H., Byers, A. y Price, L. (2007). The co-occurrence of adolescent boys' and girls' use of psychologically, physically, and sexually abusive behaviours in their dating relationships. Journal of Adolescence, 30(3), 487-504. 
Serrano, R. (2013). Los mexicanos: Reprobados en educación sexual. Salud y Medicinas, Centro de Salud Femenina. Recuperado de <http://www. saludymedicinas.com.mx/centros-de-salud/salud-femenina/articulos/losmexicanos-reprobados-en-educacion-sexual.html $>$.

Servicios a la Juventud. (Sin año). Observatorio de Violencia Social, Género y Juventud, Servicios a la Juventud. Recuperado de <http://observatorio.seraj.org.mx/ index.php?option $=$ com_content\&view $=$ article\&id $=62 \&$ Itemid $=5>$.

Tolan, P., Gorman-Smith, D. y David, H. (2006). Family violence. Annual Review of Psychology, 57(1), 557-583.

Treviño, G. (2012). El género y la violencia en la pareja. En L. Niño, L. Ovalle, R. Balbuena e I. González (Coords.), Los estudios de género en las fronteras de la violencia: Enfoques teóricos y metodológicos, reflexiones, experiencias y políticas públicas, (pp. 89-94). Mexicali, Baja California: Universidad Autónoma de Baja California.

Trujillo, M., Anda, J. de, Montoya, M. (2012). Proyecto educativo para la detección, prevención y atención de la violencia contra las mujeres (Pedpavim) en el Instituto Tecnológico de Aguascalientes (ITA), México. Perspectivas Sociales, 13(2), 1-17.

Trujano, P., Nava, C., Tejeda, E. y Gutiérrez, G. (2006). Estudio confirmatorio acerca de la frecuencia y percepción de la violencia: El vidofyp como instrumento de evaluación y algunas reflexiones psicosociales. Intervención Psicosocial, 15(1), 95-110.

Valdez-Santiago, R., Torres, M., Garda, R., Gómez, C. y Quiroz, J. (2008). Programas de intervención para la violencia de pareja en México: Antecedentes y principales modelos de atención a mujeres maltratadas y hombres agresores. En M. Híjar y R. Valdez-Santiago, (Eds.), Programas de intervención con mujeres víctimas de violencia de pareja y con agresores. Experiencia internacional y mexicana (pp. 45-69). México: Instituto Nacional de Salud Pública.

Vázquez, S., Grajeda, M. y Domínguez, M. (2012). Violencia de género en el noviazgo en adolescentes. En L. Niño, L. Ovalle, R. Balbuena e I. González (Coords.), Los estudios de género en las fronteras de la violencia: Enfoques teóricos y metodológicos, reflexiones, experiencias y políticas públicas (pp. 619-635). Mexicali, Baja California: Universidad Autónoma de Baja California.

Velázquez, G. (2011). La violencia durante el noviazgo en adolescentes. Elementos: Ciencia y Cultura, 19(82), 39-43. Recuperado de < http://www.elementos.buap. $\mathrm{mx} / \mathrm{num} 82 / \mathrm{pdf} / 39 . \mathrm{pdf}>$.

Zamudio, F., Ayala, M. y Andrade, M. (2011). Violencia en el alumnado de la Universidad Autónoma Chapingo, según género. Memorias del XI Congreso Nacional de Investigación Educativa, Universidad Nacional Autónoma de México, México. 